\title{
THE EFFECT OF TEMPERATURE PERTURBATIONS ON ION-ACOUSTIC AND DRIFT WAVES IN A WEAKLY COLLISIONAL PLASMA
}

\author{
R. S. B. ONG and M. Y. YU \\ Department of Aerospace Engineering, The University of Michigan, Ann Arbor, \\ Mich. 48104, U.S.A.
}

(Received 29 June 1972; and in final form 25 October 1972)

\begin{abstract}
The influence of temperature perturbations on the propagation and stability of low frequency waves in a weakly collisional plasma is analyzed. Temperature oscillations in general reduce the stability of ion acoustic waves. It also tends to lower the magnitude of the critical current necessary for triggering the instability. In the case of drift waves its effect is to change the destabilizing role of electron-ion collisions in the isothermal model into a damping influence.
\end{abstract}

\section{INTRODUCTION}

THE EFFECT of weak collisions on the propagation and stability of low frequency waves in a fully ionized plasma is often investigated by means of kinetic equations with an appropriate collison operator. In order to properly account for the dominant small angle collisions the Fokker-Planck collision operator is frequently used. However, the complete Fokker-Planck kinetic equation (MONTGOMERY and TIDMAN, 1964) is mathematically extremely complicated and very often one has to resort to numerical methods. To make the analysis tractable a model Fokker-Planck operator is very frequently employed (DOUGHERTY, 1964; OPPENHEIM, 1965). This model also incorporates a diffusion in velocity space and hence the dominant effect of small angle interactions is to a certain extent correctly taken into account. However, the model omits the details of the collision mechanism such as the velocity dependence of the collision frequencies and the anisotropy of the dynamical friction force. Instead effective collision frequencies must be specified a priori (ONG and YU, 1969, 1970).

In the model Fokker-Planck collision operator the electron and ion temperatures are usually assumed constant, i.e. the plasma is assumed isothermal. This approximation has the simplifying consequence that the coefficient of the diffusion term in the collision operator is a constant. It has been noted recently that temperature perturbations may have a significant infuence on the stability criteria of low frequency waves in collision dominated plasmas (ROGNLIEN and SELF, 1971). In this paper we shall study the effect of temperature perturbations on the propagation and stability of ionacoustic and drift waves in a weakly collisional plasma with $T_{i} \approx T_{e}$. The electron and ion temperatures will be allowed to vary. Consequently, the velocity space diffusion term in the model Fokker-Planck collision operator now has a variable coefficient. The variation in the temperatures will have to be determined through the macroscopic conservation equations.

The kinetic equations for ions and electrons are solved by means of the usual perturbation technique. Instead of applying the traditional method of integration along particle trajectories, we employ a velocity space Fourier transform (ONG and $\mathrm{YU}, 1970$ ). This method is preferable because of the relatively complicated nature of the model Fokker-Planck collision terms. The dispersion relation and growth rate are then determined for the case of small collision frequencies $\left(v / k_{\|} v_{T i} \ll 1, \nu / \Omega_{i} \ll 1\right.$, $\nu / \omega \ll 1)$ and the stability of the oscillations investigated. 
Our results show that temperature perturbations in general reduce the stabilizing factors in the case of ion-acoustic waves. It also tends to decrease the electron current necessary for instability. In the case of the drift-wave or universal instability, the effect of temperature fluctuations yields stabilization by electron-ion collisions, while the iso-thermal calculations predict instability. Similar results are also obtained in a recent investigation of the drift wave instability by BHADRA (1971), who solved the the complete Fokker-Planck kinetic equation numerically, and obtained electron-ion collisional damping for very small collisional frequencies.

\section{FORMULATION OF THE PROBLEM}

A low- $\beta$ singly ionized plasma is immersed in a stationary externally applied magnetic field directed along the $z$-axis of a Cartesian coordinate system. The plasma density and temperature may vary in the $x$-direction. We assume that a temperature difference between electrons and ions is maintained. This may be accomplished by introducing an external heat source for the electrons and an external heat sink for the ions, as is often found in laboratory discharges. *

We consider low-frequency electrostatic waves of the form $\exp (i \mathbf{k} \cdot \mathbf{r}-i \omega t)$, where $\mathbf{k}=k_{y} \mathbf{e}_{y}+k_{\|} \mathbf{e}_{z}$. The kinetic equations for the electrons and ions are

$$
\frac{\partial f_{j}}{\partial E}+\mathbf{v} \cdot \frac{\partial f_{j}}{\partial \mathbf{r}}+\frac{e_{j}}{m_{j}}\left(\mathbf{E}+\frac{1}{c} \mathbf{v} \times \mathbf{B}\right) \cdot \frac{\partial f_{j}}{\partial \mathbf{v}}=J_{j j}\left(f_{j}\right)+J_{j l}\left(f_{j}\right)
$$

where

$$
\begin{aligned}
& J_{j j}\left(f_{j}\right)=v_{j j} \frac{\partial}{\partial \mathbf{v}} \cdot\left[\frac{T_{j}}{m_{j}} \frac{\partial}{\partial \mathbf{v}}+\left(\mathbf{v}-\mathbf{u}_{j}\right)\right] f_{j} \\
& J_{j l}\left(f_{j}\right)=v_{j l} \frac{\partial}{\partial \mathbf{v}} \cdot\left[\frac{T_{j}}{m_{j}} \frac{\partial}{\partial \mathbf{v}}+\left(\mathbf{v}-\frac{v_{l j} m_{l}}{v_{j l} m_{j}} \mathbf{u}_{j}\right)\right] f_{j} .
\end{aligned}
$$

The subscripts $j$ and $l$ denote either electrons or ions.

The number density, mean velocity and temperature are given respectively by

$$
\begin{aligned}
n_{j}(\mathbf{r}, t) & =\int_{-\infty}^{\infty} f_{j} \mathrm{~d}^{3} \mathbf{v} \\
n_{j} \mathbf{u}_{j}(\mathbf{r}, t) & =\int_{-\infty}^{\infty} \mathbf{v}_{j} f_{j} \mathrm{~d}^{3} \mathbf{v} \\
3 n_{j} T_{j}(\mathbf{r}, t) & =m_{j} \int_{-\infty}^{\infty}\left(\mathbf{v}_{j}-\mathbf{u}_{j}\right) f_{j} \mathrm{~d}^{3} \mathbf{v} .
\end{aligned}
$$

The terms $J_{j j}$ and $J_{j l}$ represent collisions between like and unlike particles respectively. The collision operators satisfy conservation of number, momentum and energy in like particle collisions. They also satisfy conservation of number and total momentum in unlike particle collisions. Total energy is conserved in unlike particle collisions only if there is no external source of energy, such as, for example, an applied electric field. Otherwise, we must assume that energy is dissipated by radiation through unlike particle collisions. In this way a "steady state" can still be maintained provided the applied electric field is sufficiently small.

* The authors thank the Referee for his suggestions in regard to this matter. 
We also have the equations of continuity, the equations of conservation of momentum, and Poisson's equation. These may be written in the form:

$$
\begin{aligned}
\frac{\partial n_{j}}{\partial t}+\frac{\partial}{\partial \mathbf{r}} \cdot\left(n_{j} \mathbf{u}_{j}\right) & =0 \\
m_{j} n_{j}\left(\frac{\partial \mathbf{u}_{j}}{\partial t}+\mathbf{u}_{j} \cdot \frac{\partial \mathbf{u}_{j}}{\partial \mathbf{r}}\right) & =-\nabla\left(n_{j} T_{j}\right)+\nabla . \overline{\bar{\pi}}_{j}+n_{j} e_{j}\left(\mathbf{E}+\frac{1}{c} \mathbf{u}_{j} \times \mathbf{B}\right) \\
\nabla \cdot \mathbf{E} & =4 \pi \sum_{j} e_{j} n_{j}
\end{aligned}
$$

where $\overline{\bar{\pi}}_{j}$ is the traceless stress tensor.

We linearize equation (1) and carry out a normal mode analysis assuming the disturbances to be of the form $\exp (i \mathrm{k} \cdot \mathbf{r}-i \omega t)$. Throughout the analysis we use the assumption that collisions are weak. This implies that the largest collision frequency of the particles in the plasma is very small compared with either the wave frequency or the ion Larmor frequency. The steady state kinetic equation is satisfied to order $\nu / \Omega_{i}$ by the following velocity distribution which is nearly Maxwellian:

where

$$
f_{0 j}(\mathbf{r}, \mathbf{v})=\left\{1+\left(x+\frac{v_{y}}{\Omega_{j}}\right)\left[\varepsilon_{j}-\frac{3}{2} \delta_{j}+\frac{m_{j} \delta_{j}}{2 T_{j}}\left(\mathbf{v}-\mathbf{u}_{j 0}\right)^{2}\right]\right\} f_{M j}(\mathbf{v})
$$

$$
\begin{aligned}
f_{M j}(\mathbf{v}) & =n_{j}\left(m_{j} / 2 \pi T_{j}\right)^{3 / 2} \exp \left\{-\frac{m_{j}}{2 T_{j}}\left(\mathbf{v}-\mathbf{u}_{j 0}\right)^{2}\right\} \\
\varepsilon_{j} & =\frac{1}{n_{j}} \frac{\mathrm{d} n_{j}}{\mathrm{~d} x} \\
\delta_{j} & =\frac{1}{T_{j}} \frac{\mathrm{d} T_{j}}{\mathrm{~d} x} \\
\Omega_{j} & =\frac{e_{j} B}{m_{j} c} .
\end{aligned}
$$

The drift velocity $\mathbf{u}_{j 0}$ may be related to the external electric field $\mathbf{E}_{0}$. If the drift velocity $\mathbf{u}_{i 0}$ of the ions is taken to be zero, then the corresponding electron drift velocity is given by

$$
\mathbf{u}_{e 0}=-e \mathrm{E}_{0} / m_{e} v_{e i}
$$

It may then be shown that the resulting Ohmic heating is exactly equal to the total energy loss in the unlike particle collisions (ONG and YU, 1969).

We combine the like and unlike particle collision terms in the linearized first order equations and obtain

$$
\begin{aligned}
\left(i \omega-i \mathbf{k} \cdot \mathbf{v}+3 v_{j}\right) f_{j 1}-\left\{\frac { e _ { j } } { m _ { j } } \left(\mathbf{E}_{0}+\right.\right. & \left.\left.\frac{1}{c} \mathbf{v} \times \mathbf{B}_{0}\right)-v_{j}\left(\mathbf{v}-\mathbf{u}_{j 0}^{*}\right)\right\} \cdot \frac{\partial f_{j}}{\partial \mathbf{v}}+\frac{v_{j} T_{j 0}}{m_{j}} \frac{\partial^{2} f_{j 1}}{\partial v^{2}} \\
& =\left(\frac{e_{j}}{m_{j}} \mathbf{E}_{1}+v_{j} \mathbf{u}_{j 1}^{*}\right) \cdot \frac{\partial f_{j 0}}{\partial \mathbf{v}}+\frac{v_{j} T_{j 1}}{m_{j}} \frac{\partial^{2} f_{j 0}}{\partial v^{2}}
\end{aligned}
$$

where

$$
\begin{aligned}
\mathbf{u}_{j}^{*} & =\frac{1}{v_{j}}\left(v_{j j} \mathbf{u}_{j}+v_{j l} \frac{m_{l} n_{l}}{m_{j} n_{j}} \mathbf{u}_{l}\right) \\
v_{j} & =v_{j j}+v_{j l}
\end{aligned}
$$


the last term in (7) is due to the temperature perturbation, which contributes only to the non-homogeneous part of the equation for $f_{j l}$.

To solve equation (7) we apply a Fourier transformation in velocity space:

$$
G_{j}(\sigma)=\int_{-\infty}^{\infty} f_{j 1}(\mathbf{v}) \exp (-i \sigma \cdot \mathbf{v}) \mathrm{d}^{3} \mathbf{v}
$$

The perturbation number density, $n_{j l}$, is then given by $G_{j}(0)$. The transformed equation may be rearranged into the form:

where

$$
a(\boldsymbol{\sigma}) G(\boldsymbol{\sigma})+\mathbf{b}(\boldsymbol{\sigma}) \cdot \frac{\partial}{\partial \boldsymbol{\sigma}} G(\boldsymbol{\sigma})=c(\boldsymbol{\sigma})
$$

$$
\begin{aligned}
a(\boldsymbol{\sigma})= & -i \omega+i \nu u_{0} \sigma_{z}+\frac{\nu T \sigma^{2}}{m} \\
\mathbf{b}(\boldsymbol{\sigma})= & -\mathbf{k}+\nu \boldsymbol{\sigma}+\boldsymbol{\sigma} \times \Omega \\
c(\boldsymbol{\sigma})= & -n_{0}\left(\frac{e}{m} \mathbf{E}_{1}+\nu \mathbf{u}_{1}^{*}-i \frac{\nu T_{1}}{m} \boldsymbol{\sigma}\right) \\
& \times\left[i \boldsymbol{\sigma}+\frac{\mathbf{e}_{y}}{\Omega}\left(\varepsilon+\frac{1}{2} \delta \frac{T_{1}}{m} \sigma^{2}\right)\right] \exp \left(-i u_{0} \sigma_{z}-\frac{T \sigma^{2}}{2 m}\right)
\end{aligned}
$$

The subscripts which indicate the various species have been omitted. Equation (9) is a first order partial differential equation and its solution may be obtained by the method of characteristics. In terms of the parameter $t$ the characteristic equations are

$$
\begin{gathered}
\frac{\mathrm{d} \sigma^{\prime}(t)}{\mathrm{d} t}=\mathbf{b}\left[\boldsymbol{\sigma}^{\prime}(t)\right] ; \quad \sigma^{\prime}(t=0)=\sigma \\
a\left(\boldsymbol{\sigma}^{\prime}\right) G\left(\sigma^{\prime}\right)+\frac{\mathrm{d}}{\mathrm{d} t} G\left(\boldsymbol{\sigma}^{\prime}\right)=c\left(\boldsymbol{\sigma}^{\prime}\right) .
\end{gathered}
$$

We can integrate (11) and obtain

$$
G\left(\boldsymbol{\sigma}^{\prime}\right)=\int_{-\infty}^{t} \exp \left\{\int_{t}^{t^{\prime}} a\left[\boldsymbol{\sigma}^{\prime}\left(t^{\prime \prime}\right)\right] \mathrm{d} t^{\prime \prime}\right\} c\left[\boldsymbol{\sigma}^{\prime}\left(t^{\prime}\right)\right] \mathrm{d} t^{\prime} .
$$

The vector $\sigma^{\prime}(t)$ is then determined by its components which are given by the solutions of $(10)$

$$
\begin{aligned}
\sigma_{x}^{\prime}(t)= & -\left(k_{y} \Omega / \Omega^{2}+\nu^{2}\right)+\exp (\nu t)\left\{\left(\sigma_{x}+\frac{k_{y} \Omega}{\Omega^{2}+\nu^{2}}\right) \cos \Omega t\right. \\
& \left.+\left(\sigma_{y}-\frac{k_{y} \Omega}{\Omega^{2}+v^{2}}\right) \sin \Omega t\right\} \\
\sigma_{y}^{\prime}(t)= & \left(k_{y} \Omega / \Omega^{2}+\nu^{2}\right)+\exp (v t)\left\{\left(\sigma_{y}-\frac{k_{y} \Omega}{\Omega^{2}+\nu^{2}}\right) \cos \Omega t\right. \\
& \left.-\left(\sigma_{x}+\frac{k_{y} \Omega}{\Omega^{2}+v^{2}}\right) \sin \Omega t\right\} \\
\sigma_{z}^{\prime}(t)= & \left(k_{\|} / \nu\right)+\left(\sigma_{z}-\frac{k_{\|}}{\nu}\right) \exp (v t) .
\end{aligned}
$$


It is to be noted that (12) is a rather complicated but exact integral of the linearized kinetic equation (7). In the limit of small collision frequencies we neglect terms of order $\left(v_{j} / \Omega_{j}\right)^{2}$ and $\left(v_{j} / k_{i j} v_{T j}\right)^{2}$, with $v_{T j}=\left(T_{j} / m_{j}\right)^{1 / 2}$, the thermal velocity of the particle of species $j$. From the relation $n_{1}=G(0)$ we then find the perturbation number density. This is given by

where

$$
\begin{aligned}
n_{1}= & -\frac{1}{T}\left[n_{0} e \phi_{1} \exp (-b)\right] \sum_{l}\left\{A_{0}+i \nu A_{1}-i \nu \omega * A_{2}-i \nu \omega^{*} b \eta A_{3}\right\} \\
& -i n_{0} \nu \exp (-b) \sum_{l}\left\{A_{4}+\frac{T_{1}}{T_{0}} A_{5}\right\}
\end{aligned}
$$

$$
\begin{aligned}
i \mathbf{k} \phi_{1} & =\mathbf{E}_{1} \\
C & =\frac{1}{2}\left(k_{y} v_{T} / \Omega\right)^{2} \\
\eta & =\delta / \epsilon \\
\omega^{*} & =c k_{y} T \epsilon / e B .
\end{aligned}
$$

Furthermore, in equation (14), we have made the frequencies and velocities dimensionless by dividing them by $k_{\|} v_{T}$ and $v_{T}$ respectively. The functions $A_{i}$ with $i=$ $0,1, \ldots, 5$ are given by the following expressions:

$$
\begin{aligned}
A_{0}= & l \Omega I_{l} Z_{l}-\frac{1}{2} I_{l} Z_{l}^{\prime}-\omega *\left[(1+b \eta) I_{l} Z_{l}-b \eta I_{l} Z_{l}-\frac{1}{4} \eta I_{l} Z_{l}^{\prime \prime}\right] \\
A_{1}= & {\left[(2 l+b) I_{l}^{\prime}-2 l I_{l} Z_{l}-\frac{1}{2} b I_{l}^{\prime} Z_{l}^{\prime \prime}-\frac{1}{24} I_{l} Z_{l}^{I V}\right.} \\
& +\Omega I_{l}\left[(2-b) l Z_{l}^{\prime}-\frac{1}{4} Z_{l}^{\prime \prime}-\frac{1}{12} l Z_{l}^{\prime \prime}\right] \\
A_{2}= & -b I_{l} Z_{l}^{\prime}+\frac{2 l}{\Omega} I_{l} Z_{l}-\frac{1}{12} I_{l} Z_{l}^{\prime \prime} \\
A_{3}= & {\left[b\left(I_{l}-I_{l}^{\prime}\right)+\frac{l^{2}}{b} I_{l}-I_{l}^{\prime}\right] Z_{l}^{\prime}+\frac{1}{6}\left(\frac{1}{2} I_{l}+I_{l}^{\prime}\right) Z_{l}^{\prime \prime \prime}+\frac{1}{4} \overline{8} I_{l} Z_{l}^{\nabla} } \\
& +\frac{1}{\Omega}\left[\frac{l}{2 b} I_{l} Z_{l}^{\prime \prime}-2 l\left(I_{l}-I_{l}^{\prime}+\frac{1}{b} I_{l}\right) Z_{l}\right] \\
A_{4}= & -i \frac{k_{y}}{k_{l \mid} \Omega}\left(I_{l}-I_{l}^{\prime}\right) Z_{l} u_{i x}{ }^{*}-\frac{k_{y}}{k_{\|} \Omega}\left\{\left(\frac{i}{b}+\frac{\varepsilon}{k}\right) I_{l} Z_{l}\right. \\
& \left.+\frac{\delta}{k_{y}}\left[b\left(I_{l}-I_{l}^{\prime}\right) Z_{l}-\frac{1}{4} I_{l} Z_{l}^{\prime \prime}\right]\right\} u_{1 y}{ }^{*}-I_{l} Z_{l}^{\prime} u_{1 z}{ }^{*} \\
A_{5}= & 2 b\left(I_{l}-I_{l}^{\prime}\right) Z_{l}-\frac{1}{4} I_{l} Z_{l}^{\prime \prime}-i \frac{\varepsilon}{k_{y}} l I_{l} Z_{l} \\
& -\frac{\delta}{k_{y}} l\left\{\left[(1+b) I_{l}-b I_{l}^{\prime}\right] Z_{l}-I_{l} Z_{l}^{\prime \prime}\right\}
\end{aligned}
$$

$I_{l} \equiv I_{l}(b)$ is the Bessel function of order $l$ with imaginary argument. $Z_{l} \equiv Z_{l}(\omega-$ $k_{\|} u_{0}+i b v-l \Omega$ ) is the plasma dispersion function (ONG and YU, 1969, 1970). The superscripts on $I_{l}$ and $Z_{l}$ denote derivatives.

The quantities $u_{1}{ }^{*}$ and $T_{1}$ are related to $n_{1}$ and $\phi_{1}$ through the continuity and 
momentum equations (3) and (4) as follows:

$$
\begin{aligned}
\mathbf{u}_{j 1}^{*} & =-i \frac{\omega}{n_{j 0} k^{2} \nu_{j}}\left(\varepsilon \mathbf{e}_{x}+i \mathbf{k}\right)\left(v_{j j} n_{j 1}+\frac{m_{j}}{m_{l}} v_{l j} n_{l 1}\right) \\
T_{j 1} & =-\frac{T_{j 0}}{n_{j 0}} n_{j 1}-e \phi_{j 1}+\frac{m_{j}}{k_{\|}}\left(\omega-k_{11} u_{j 0}-k_{y}(1+\eta) \frac{c T_{j} \varepsilon_{j}}{e_{j} B}\right) u_{j 1 x} .
\end{aligned}
$$

As we are restricting ourselves to weak collisional effects the contribution of the traceless stress tensor to the expression for $T_{j l}$ in (16) is negligible since this would lead to terms of order $\left(v_{j} / \Omega_{j}\right)^{2}$ and $\left(v_{j} / k_{\| j} v_{T j}\right)^{2}$ in the dispersion relation.

We may now readily obtain the dispersion relation by the substitution of equations (14), (15) and (16) into Poisson's equation (5).

\section{LOW FREQUENCY WAVES}

In this section we consider electrostatic waves with frequencies much less than the ion cyclotron frequency. In particular, the ion-acoustic and drift waves will be investigated. The former may be unstable in the presence of a drift current parallel to the magnetic field. The latter may be unstable if finite Larmor radius effects are significant, or when there are sufficiently large gradients in the plasma. As we are considering the case where $\omega \ll \Omega_{i}$, only the zero ${ }^{t h}$ order Bessel functions contribute to the summation in (14).

(a) Ion-acoustic wave instability

In considering the ion-acoustic wave instability, we shall neglect the inhomogeneity effects. Moreover, we may assume

$$
V_{T i} \ll \omega / k_{\mathrm{ii}} \ll V_{\bar{T} e} .
$$

We now let $\omega=\omega_{0}+i \gamma$, and assume $\gamma \ll \omega_{0}$. The real part of the dispersion relation yields the ion-acoustic wave frequency

$$
\omega_{0}=k_{\| l}\left(T_{e}\left(m_{i}\right)^{1 / 2}\left(1+k^{2} \lambda_{e}^{2}\right)^{-1 / 2} ; \quad k \lambda_{e}<1 .\right.
$$

where $\lambda_{e}$ is the electron Debye length.

The imaginary part of the dispersion relation gives the growth rate of the instability. This can be written as

where

$$
\gamma=\gamma_{0}+\gamma_{i}+\gamma_{e}
$$

$$
\begin{aligned}
\gamma_{0} \simeq & -\frac{1}{2}\left(\pi m_{e} / m_{i}\right)^{1 / 2}\left(\omega_{0}-k_{\|} u_{e 0}\right)\left(1+k \lambda_{e}^{2}\right)^{-1 / 2} \\
& -\pi^{1 / 2}\left(\omega_{0}^{4} / k_{\|}^{3} v_{T i}^{3}\right) \exp \left(-\omega_{0}^{2} / k_{\|}^{2} v_{T i}^{2}\right) \\
\gamma_{i} \simeq & -\frac{1}{2} v_{i i} \\
\gamma_{e} \simeq & \left(m_{e} / m_{i}\right) v_{e i}\left[1-\left(k_{\|} u_{e 0} / \omega_{0}\right)\right]\left[\frac{1}{2}+\frac{T_{i}}{T_{e}} k^{2} \lambda_{e}^{2}-\frac{19}{6}\left(1+k^{2} \lambda_{e}^{2}\right)^{-2}\right] .
\end{aligned}
$$

Here $\gamma_{0}, \gamma_{i}$ and $\gamma_{e}$ represent the attenuation due to wave-particle interactions, ionion collisions and electron-ion collisions respectively. Note that as far as collisional effects are concerned drift is coupled via the electron-ion collisions in a destabilizing sense if $k_{k} u_{e 0} / \omega_{0}>0$, i.e. for the wave with phase velocity in the sense of $u_{e 0}$. It is seen that ion-ion collisions always lead to a damping effect. However, in the presence of a drift current, electron-ion collisions may increase the growth rate of the instability. 
In the isothermal case the coefficient $\frac{19}{6}$ in $\gamma_{e}$ is replaced by $\frac{49}{6}$. This shows that the effect of the temperature perturbations is to lower the stabilizing influence of the electron-ion collisions.

The critical electron drift velocity above which the plasma becomes unstable is

$$
\begin{aligned}
u_{e 0} \simeq & \omega_{0} / k_{\|}+\pi^{1 / 2}\left(m_{i} / m_{e}\right)\left(v_{i i} / k_{\|}\right)\left(1+k^{2} \lambda_{e}{ }^{2}\right)^{2} \\
& +\left(m_{i} / m_{e}\right)^{1 / 2}\left(T_{e} / T_{i}\right)^{3 / 2}\left(\omega_{0} / k_{\|}\right) \exp \left(-\omega_{0}^{2} / k_{\|}^{2} v_{T i}^{2}\right) \\
& -\frac{8}{\pi}\left(m_{i} / m_{e}\right)^{1 / 2}\left(T_{e} / T_{i}\right)^{3 / 2}\left(v_{e i} / k_{\|}\right)\left[\frac{1}{2}+k^{2} \lambda_{e}{ }^{2} T_{i} / T_{e}-\frac{19}{6}\left(1+k^{2} \lambda_{e}{ }^{2}\right)^{-2}\right] \\
& \times\left(1+k^{2} \lambda_{e}{ }^{2}\right)^{4} \exp \left(-\omega_{0}{ }^{2} / k_{\|}{ }^{2} v_{T i}{ }^{2}\right) .
\end{aligned}
$$

Equation (18) indicates that temperature perturbations tend to reduce the magnitude of the critical electron drift velocity necessary for instability. Ion-ion collisions again shows a stabilizing infuence. In the isothermal limit the expressions for $\omega_{0}$ and $u_{e 0}$ above are quantitatively slightly different from our earlier results (ONG and YU, 1969) because here we have neglected terms of order higher than $\left(k_{\| !} V_{T i} / \omega\right)^{2}$, while in the earlier paper such terms are included in the analysis via interaction. In Fig. 1 we show the effect of temperature perturbation on the threshold drift $u_{e 0}$ in the case of an ion acoustic wave as $k \lambda_{e}$ and $T_{i} / T_{e}$ are varied. The growth rates are shown in Fig. 2.

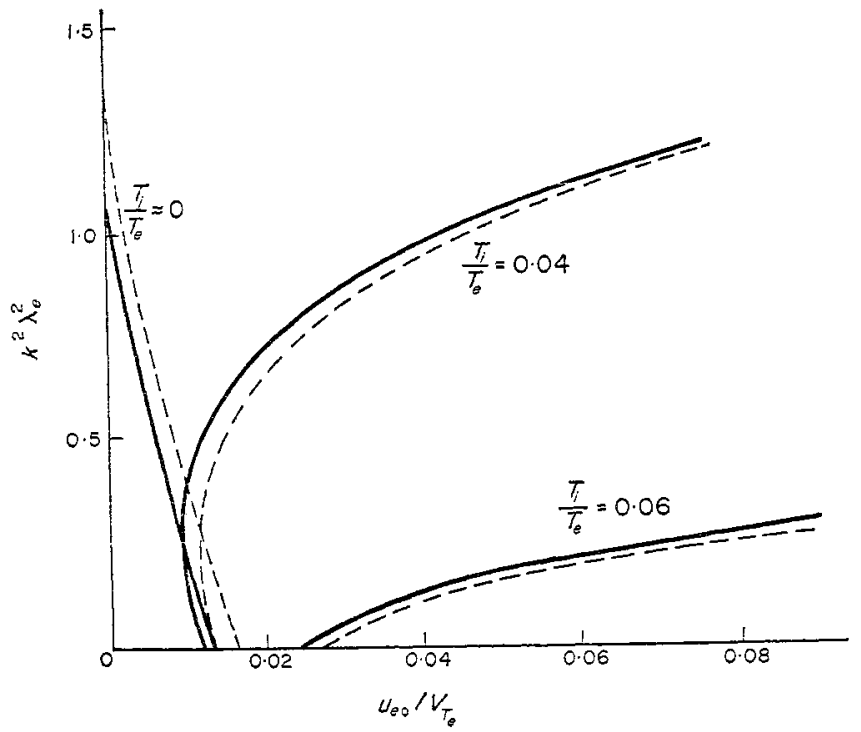

FIG. 1.-The threshold current of the ion-acoustic wave instability. Results from isothermal calculations are indicated by the dashed curves. The regions to the right of the curves are unstable.

(b) Drift wave instability

To consider the drift wave or universal instability we retain the inhomogeneity effects while neglecting the external electron drift current. Again, we shall assume the wave phase velocity ordering as given by (17). For the propagation frequency of the drift wave we obtain

$$
\omega_{0}=\omega_{e}{ }^{*} \xi_{0} /\left(2+k^{2} \lambda_{i}{ }^{2}-\xi_{0}\right) ; \quad k \lambda_{i}=k v_{T i} / \omega_{p i} \ll 1
$$




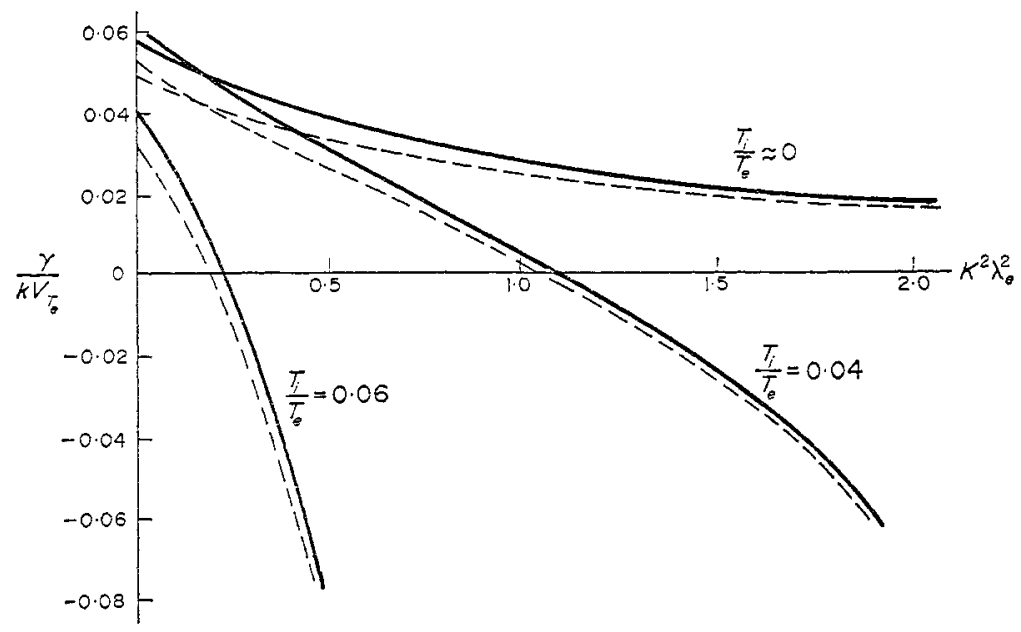

FIG. 2.-The growth rate of the ion-acoustic instability.

where we have let $T_{i}=T_{e}, b_{e}=0, \mathrm{~d} T / \mathrm{d} x=0$ and $\xi_{0}(b)=I_{0}(b) \exp (-b)$ with $b \equiv b_{i}=\frac{1}{2}\left(k_{y} v_{T_{i}} / \Omega_{i}\right)^{2}$. From the imaginary part of the dispersion relation we obtain the growth rate for the case where $b \ll 1$, i.e. $k_{y}{ }^{2} r_{L i}{ }^{2} \ll 1$, where $r_{L i}$ is the ion Larmor radius:

$$
\begin{aligned}
\gamma_{0} \simeq & \left(m_{e} / m_{i}\right)^{1 / 2} \omega_{0}{ }^{2}\left(1+b+k^{2} \lambda_{i}^{2}\right)^{-1} \\
& \times\left\{\pi^{1 / 2}\left(2 b+k^{2} \lambda_{i}^{2}\right)(1-b)^{-1}-\pi^{1 / 2}\left(m_{i} / m_{e}\right)^{1 / 2}\left(2+k^{2} \lambda_{i}^{2}\right) \exp \left(-\omega_{0}^{2}\right)\right\} \\
\gamma_{i} \simeq & -v_{i i}\left(1+b+k^{2} \lambda_{i}^{2}\right)^{-1}\left\{2 b+\frac{1}{2}(1+7 b) k^{2} \lambda_{i}^{2}\right. \\
& \left.+2 \frac{k_{\|}^{2}}{k^{2}}\left(1+k^{2} \lambda_{i}^{2}\right) \omega_{0}^{2}\left[1+k^{2} \lambda_{i}^{2}+\frac{1}{2} b\left(7-k^{2} \lambda_{i}^{2}\right)\right]\right\} \\
\gamma_{e} \simeq & \left(m_{e} / m_{i}\right) v_{e i} \omega_{0}^{2}\left(1+b+k^{2} \lambda_{i}^{2}\right)^{-1} \\
& \times\left\{-4+\frac{4}{3}\left[2 b+(1+b) k^{2} \lambda_{i}^{2}\right]-2 \frac{k_{\|}{ }^{2}}{k^{2}}\left[\frac{1}{2} b\left(3-k^{2} \lambda_{i}^{2}\right)+k^{2} \lambda_{i}^{2}\right]\right\}
\end{aligned}
$$

In the expressions above the frequencies are non-dimensionalized with respect to $k_{\| i} v_{T i}$. In the isothermai case the terms $2 b, 1+7 b$ and $7-k^{2} \lambda_{i}{ }^{2}$ in the expression for $\gamma_{i}$ are replaced by $4 b, 1+3 b$ and $3-k^{2} \lambda_{i}{ }^{2}$ respectively. More important, the large damping term -4 in $\gamma_{e}$ does not appear in the result for the isothermal case. Figure 3 shows these results.

Consequently, we note that the isothermal assumption leads to an overestimation of the ion-ion collisional damping. On the other hand, the inclusion of temperature perturbations changes the effect of electron-ion collisions from growth to damping. The latter result has also been found by BHADRA (1971), who solved the full FokkerPlanck equation numerically for the effect of electron-ion collisions on the universal instability.

In the limit of weak collisions Bhadra's result (equation (18) in his article) can be written in the form

$$
\gamma_{e} \sim-\frac{4}{3 \pi^{1 / 2}}\left(m_{e} / m_{i}\right) v_{e i} b
$$




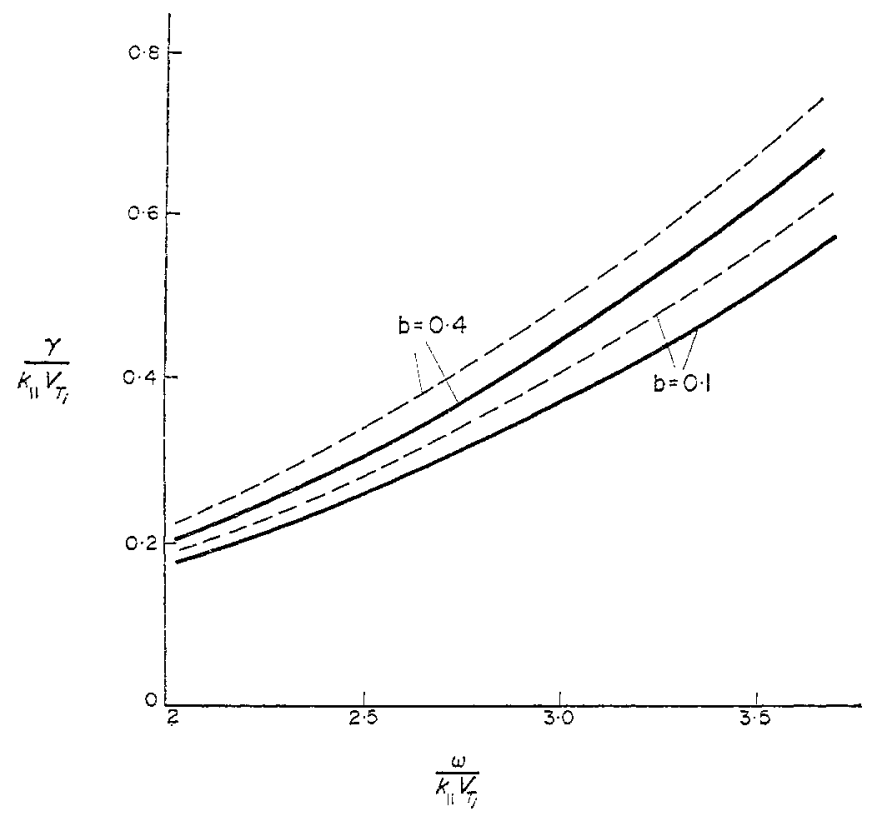

FIG. 3.-The growth rate of the drift wave instability.

This is to be compared with the most significant term in our expression for $\gamma_{e}$ above, namely

$$
\gamma_{e} \sim-4\left(m_{e} / m_{i}\right) \nu_{e i} b k_{\|}{ }^{-2}\left(\frac{1}{n} \frac{\mathrm{d} n}{\mathrm{~d} x}\right)^{2} .
$$

The growth rate for $b>1$ is given by

$$
\begin{aligned}
\gamma_{0} \simeq & \left(m_{e} / m_{i}\right)^{1 / 2} \omega_{0}{ }^{2}\left[\pi(2 b)^{1 / 2}-\pi^{1 / 2}\left(m_{i} / m_{e}\right) \exp \left(-\omega_{0}{ }^{2}\right)\right. \\
\gamma_{i} \simeq & -\nu_{i i} \omega_{0}{ }^{-2}\left(b m_{i} / 2 \pi m_{e}\right)^{1 / 2}\left[\pi+\left(2+k^{2} \lambda_{i}^{2}\right)^{-1}\right] \\
& -4\left(k_{\|}{ }^{2} / k^{2}\right)\left(m_{i} / m_{e}\right)^{1 / 2} v_{i i}\left(1+k^{2} \lambda_{i}^{2}\right)\left(2+k^{2} \lambda_{i}{ }^{2}\right)^{-1} \\
\gamma_{e} \simeq & 4\left(m_{e} / m_{i}\right) v_{e i} \omega_{0}^{2}\left[\frac{2}{3}(2 \pi b)^{1 / 2}-\left(2+k^{2} \lambda_{i}{ }^{2}\right)^{-1}\right] .
\end{aligned}
$$

Again, in the above expressions all frequencies are made dimensionless with respect to $k_{\|} v_{T i}$. In the result for the isothermal case the term $\left(2+k^{2} \lambda_{i}^{2}\right)^{-1}$ does not appear, while the expression for $\gamma_{i}$ is only slightly altered. Thus, for $b>1$ the temperature perturbations does not affect the contribution of ion-ion collisions but it again introduces electron-ion collisional damping which does not appear in the isothermal model. Naturally, our conclusions above are valid only in the case of weak collisions in the plasma.

\section{CONCLUSION}

We have studied the influence of temperature perturbations on the propagation and stability of low frequency waves in a fully ionized plasma. In regard to ion acoustic waves it reduces electron-ion collisional damping and decreases the magnitude of the drift current necessary for instability. On the other hand, temperature perturbations have the effect of reversing the destabilizing influence of electron-ion collisions found in the isothermal calculations of the drift wave instability. In this case temperature oscillations actually introduce electron-ion collisional damping. 
Acknowledgement-This work was supported by the U.S. Air Force Office of Scientific Research under Grant AF-AFOSR-72-2224.

\section{REFERENCES}

BHADRA D. K. (1971) Phys. Fluids 14, 977.

DOUGHeRTY J. P. (1964) Phys. Fluids 7, 1788.

Montgomery D. C. and Tidman D. A. (1964) Plasma Kinetic Theory. McGraw-Hill, New York.

ONG R. S. B. and Yu M. Y. (1969) J. Plasma Phys. 3, 425.

ONg R. S. B. and Yu M. Y. (1970) J. Plasma Phys. 4, 729.

OPPENHEIM A. (1965) Phys. Fluids 8, 900.

RognLIen T. D. and Self S. A. (1971) Phys. Rev. Lett. 27, 792. 